\title{
Lidil
}

Revue de linguistique et de didactique des langues

Acquisition et enseignement de la morphographie

\section{Acquérir le pluriel des noms}

Existe-t-il un effet de fréquence du nom ?

Pierre Largy, Marie-Paule Cousin et Michel Fayol

\section{CpenEdition}

\section{Journals}

Édition électronique

URL : http://journals.openedition.org/lidil/663

DOI : $10.4000 /$ lidil.663

ISSN : 1960-6052

Éditeur

UGA Éditions/Université Grenoble Alpes

\section{Édition imprimée}

Date de publication : 1 décembre 2004

Pagination : $39-54$

ISBN : 2-914176-11-2

ISSN : 1146-6480

\section{Référence électronique}

Pierre Largy, Marie-Paule Cousin et Michel Fayol, « Acquérir le pluriel des noms », Lidil [En ligne],

30 | 2004, mis en ligne le 29 janvier 2008, consulté le 02 mai 2019. URL : http://

journals.openedition.org/lidil/663; DOI : 10.4000/lidil.663 


\title{
ACQUÉRIR LE PLURIEL DES NOMS
}

\section{EXISTE-T-IL UN EFFET DE FRÉQUENCE DU NOM?}

\author{
Pierre LARGY*, Marie-Paule COUSIN ** \\ Michel FAYOL ***
}

Après avoir longtemps été dominée par des travaux sur la phonologie et la conscience phonologique, l'étude du langage, de son acquisition et de sa mise en ceuvre, porte depuis quelques années un intérêt croissant aux questions relatives à la morphologie (voir, par exemple, le numéro de la revue Rééducation Orthophonique coordonné par Marie-Pierre Thibault: «Langage écrit: morphologie et conscience morphologique», 2003). Dans ce cadre, la morphologie flexionneile du nombre a fait l'objet d'une focalisation particulière. De nombreuses recherches ont tenté d'expliquer comment la morphologie du nombre était acquise et mise en œuvre à l'oral ou à l'écrit. Ces travaux ont majoritairement concerné l'accord en nombre sujet-verbe et ont abordé de ce fait les questions relatives à la morphologie flexionnelle du nombre verbal, même si d'autres flexions verbales ont aussi retenu l'attention de certains chercheurs (par exemple en français, celles de l'imparfait «ait», du participe passé «é» ou de l'infinitif

\footnotetext{
* Université Toulouse-le-Mirail, UFR de Psychologie, 5, Allées Antonio-Machado, F-31058 Toulouse Cedex 9, <largy@univ-tlse2.fr>.

** Université de Rouen.

*** Université Blaise Pascal de Clermont-Ferrand.
} 
«er»: Brissaud, 1999a, 1999b). Une autre dimension de la morphologie flexionnelle a retenu l'intérêt des chercheurs, celle relative au nombre nominal (Totereau, Thévenin et Fayol, 1997; Totereau, Barrouillet et Fayol, 1998).

La morphologie flexionnelle du nombre nominal en français a fait l'objet de plusieurs études depuis une dizaine d'années. Celles-ci ont principalement porté sur la façon dont l'enfant au cours de son apprentissage de l'écrit la mettait progressivement en œuvre. Les chercheurs trouvaient là une excellente occasion d'étudier l'acquisition et l'utilisation par l'enfant d'une règle orthographique simple (mettre un -s à la fin d'un nom pluriel). Le français s'avérait particulièrement indiqué pour cette étude puisqu'on pourrait étudier la mise en œuvre d'une règle morphosyntaxique indépendamment d'un guidage phonologique via la flexion du nombre. En effet, le marquage de la morphologie flexionnelle du nombre, très fréquent à l'écrit en français, est principalement silencieux, qu'il s'agisse du nom (-s), de l'adjectif (-s) ou du verbe (-nt) (Dubois, 1965).

Après s'être intéressé à la compréhension par l'enfant des marques du nombre (voir Totereau, Thevenin et Fayol, 1997), les recherches ont principalement ${ }^{1}$ concerné la production de ces marques par les élèves d'école primaire. On a ainsi décrit l'acquisition de cette morphologie sous l'angle de l'apprentissage d'une règle d'accord orthographique.

Une série d'études transversales a partiellement remis en cause cette conception initiale de l'acquisition de la morphologie du nombre nominal. En effet, ces recherches ont montré qu'à différents niveaux de la scolarité primaire, tous les noms n'étaient pas accordés avec la même exactitude par le même enfant. En d'autres termes, lorsque l'enfant doit produire dans des conditions identiques l'accord de différents noms, sa performance varie en fonction des noms eux-mêmes, sans que

1. On doit toutefois préciser que parallèlement à ces recherches se sont développés depuis quelques années des travaux relatifs à la façon dont l'enfant révise des accords nominaux et verbaux et détecte des erreurs d'accords (Dédéyan et Largy, 2003; Largy, 2001 ; Largy, Chanquoy et Dédéyan, 2004; Largy, Dédéyan, 2002; Largy, Dédéyan et Hupet, sous presse). 
cette variation puisse être attribuée à la difficulté orthographique de la racine des noms. Ce résultat ne peut s'expliquer par la mise en cuvre d'un algorithme d'accord ( Si il y en a plusieurs, alors je dois mettre un $-\mathrm{s} \gg$. En effet, la production du -s par application d'un algorithme devrait s'effectuer avec la même efficacité sur tous les noms, dès lors qu'ils sont repérés comme étant des noms. Or tel n'est pas le cas puisque, longtemps au cours de sa scolarité, l'enfant accorde mieux certains noms (il accorde mieux «des cartes» que «des carpes») (Cousin, 2000).

\section{Application d'une règle ou récupération d'instances}

En lecture, certains noms peuvent être rencontrés presque exclusivement au pluriel («parents» ou «bottes») et d'autres presque exclusivement au singulier («niche» ou «pluie»). La rencontre régulière de tels noms a-t-elle un impact sur la façon dont ils seront ultérieurement fléchis en nombre par l'enfant? Ou bien l'accord en nombre du nom n'est-il régi que par la mémorisation et l'application de la règle d'accord ? En d'autres termes, une phase d'acquisition d'associations «racine nominale-flexion du nombre» précède-t-elle celle d'apprentissage de la règle d'accord? Si tel est le cas, ces acquisitions précoces interfèrent-elles ensuite avec la mise en œuvre de la règle? Une série d'expériences a récemment été conduite afin de répondre à ces questions.

Marie-Paule Cousin (2000) a testé l'hypothèse que soit l'enfant applique seulement la règle «condition - action» et, dans ce cas, tous les noms devraient être accordés de la même manière; soit il s'appuie également sur des acquisitions très localisées (sur des associations «racine nominale-flexion du nombre» mémorisées), auquel cas sa performance dans le marquage du nombre nominal devrait varier selon qu'il doit accorder des noms qu'il a fréquemment ou rarement rencontrés. Elle a proposé à des enfants et à des préadolescents de produire par écrit le seul dernier mot d'une phrase qui leur était dictée. L'auteur a ainsi pu juger de l'exactitude des accords nominaux produits. Les mots retenus étaient issus d'une base de données: le $V O B$ (Vocabulaire orthographique de base: Ters, Mayer et Reichenbach, 1969) qui permettait de 
soumettre les participants à deux types de noms à accorder, des noms censés être connus à leur niveau scolaire et des noms censés être connus à un autre niveau scolaire (en général supérieur). Les résultats montrent que les noms les plus précocement rencontrés au cours de la scolarité sont significativement mieux accordés que les noms rencontrés plus tardivement. Cet effet perdure encore en $3^{\mathrm{e}}$ de collège.

Une autre expérience (Cousin, Largy et Fayol, 2002), plus écologique, a permis d'opposer deux types d'items : des noms «effectivement» rencontrés par les enfants (dits « noms familiers ») car issus de leur propre manuel d'apprentissage de la lecture ( Gafi », 1992) et des noms «théoriquement » rencontrés issus de la base de données VOB (Ters et al., 1969) (dits « noms non-familiers») et absents du livre «Gafi». Les noms issus du $V O B$ correspondaient à la première année primaire et étaient appariés avec les noms tirés de «Gafi », en termes de nombre de lettres, nombre de syllabes et de fréquence ( $B R U$ $L E X$ : Content, Mousty et Radeau, 1990). De plus, dans la première catégorie d'items (issus de «Gafi»), les auteurs ont contrasté des noms essentiellement présentés au singulier dans cet ouvrage avec des noms qui y sont principalement rencontrés au pluriel. Comme lors de la première série d'expériences, les participants (CE1, CE2, CM1 et CM2: de la $2^{\mathrm{e}}$ à la $5^{\mathrm{e}}$ primaire) devaient écrire le dernier mot d'une phrase dictée. Deux principaux résultats émergent de cette étude et confirment les prédictions.

Premièrement, les noms effectivement rencontrés par les enfants (issus de leur manuel de lecture «Gafi») étaient mieux accordés en nombre que les noms moins familiers (items contrôles issus du $V O B$ ). Secondement, le nombre (singulier $v s$ pluriel) dans lequel les noms avaient été initialement rencontrés a une incidence sur la réalisation ultérieure de l'accord. En effet, ces résultats montrent un taux significativement plus élevé d'erreurs avec des noms «Gafi » initialement rencontrés au pluriel, qu'avec les deux autres types de noms (noms «Gafi» initialement rencontrés au singulier; noms contrôles issus du $V O B$ ), lorsqu'ils doivent être accordés au singulier.

Une nouvelle expérience (Cousin, Largy et Fayol, 2003) a été conduite avec un matériel encore plus contrasté du point 
de vue de la modalité (singulier vs pluriel) dans laquelle les noms sont le plus fréquemment rencontrés par les enfants. En effet, si l'utilisation des noms issus du manuel de lecture utilisé par les enfants a permis de confirmer l'effet de familiarité du matériel, elle permettait plus difficilement de contraster ces noms du point de vue du nombre sous lequel ils apparaissaient le plus souvent dans le livre. Les noms retenus étaient issus d'une double sélection. Une liste de noms a initialement été sélectionnée à partir de la base de données NOVLEX (Lambert et Chesnet, 2001) en retenant des noms principalement fléchis au singulier et d'autres principalement fléchis au pluriel. Cette liste a ensuite été soumise au jugement d'enseignants d'écoles primaires afin de ne retenir que les noms les plus fréquemment rencontrés par leurs élèves sous ces modalités. Les résultats obtenus lors d'une tâche de dictée identique à celle des expériences précédentes, ont permis d'étendre l'effet de la modalité initiale de rencontre à la mise au pluriel de noms initialement rencontrés au singulier. Plus précisément, les résultats de cette expérience, dans laquelle les noms sont employés de manière très contrastée soit au pluriel soit au singulier, montrent un effet significatif de ce facteur chez les enfants de CE1 et de CE2 ( $2^{e}$ et $3^{e}$ primaires). Ceux-ci accordent mieux au singulier les noms fréquemment utilisés au singulier et mieux au pluriel les noms fréquemment rencontrés au pluriel. Ces résultats confortent l'idée que la récupération d'associations «racine-flexion» interfère avec l'application de la règle.

Les recherches ici évoquées soulignent un problème essentiel, celui de mieux maitriser à l'avenir le matériel expérimental visant à tester la production de l'accord chez l'enfant, en particulier au regard de l'effet de fréquence de rencontre de ce matériel.

\section{Fréquence de rencontre et bases de données}

Si l'on souhaite étudier les effets de l'exposition quotidienne à la langue écrite sur les processus qui conduisent à son acquisition, il est nécessaire de connaitre la nature de cet input. Reconnaissons simplement que c'est impossible. Il est parfai- 
tement inimaginable de rassembler le corpus langagier auquel une personne a été exposée, comme d'ailleurs celui qu'elle a produit, sauf sur une période très limitée, ce qui en réduit considérablement l'intérêt. La question qui sera ici abordée tient davantage à la façon dont les psycholinguistes tentent de pallier cette difficulté.

Le recours au langage artificiel miniature (Miniature Artificial Language: $M A L$ ) est extrêmement fréquent dans les études sur l'acquisition de la langue maternelle (Braine et al., 1990) ou de langues étrangères (Yang et Givón, 1997). S'il est utile à l'expérimentaliste, grâce au contrôle qu'il permet sur les variables manipulées, il est très vivement critiqué dans la littérature en raison de son caractère non-écologique (Beck, 1995). Une autre façon de contourner le problème consiste à se référer à des corpus langagiers. Pour certains auteurs, il s'agit même de la seule solution: «... at best, for natural language, we can only guess by extrapolation of frequency counts from language corpora and unverifiable assumptions about registers » (Ellis et Schmidt, $1998: 310$ ).

La base de données à laquelle les psycholinguistes français ont le plus fréquemment recours est la base Brulex (Content et al., 1990), version informatisée du volumineux TLF (Trésor de la Langue française, Imbs, 1971). Toutefois, l'usage d'une telle base est problématique pour appréhender la fréquence lexicale chez l'enfant. En effet, le $T L F$ a été élaboré sur la seule base de textes littéraires (romans, essais, recueils de poèmes...) et n'est approprié, de ce fait, que pour l'adulte lettré. C'est en langue anglaise qu'on a vu naitre la première base de données lexicales établie selon différents niveaux scolaires: The educator's WFG (Zeno et al., 1995). En français, jusqu'à la récente publication de la base NOVLEX (Lambert et Chesnet, 2001), les auteurs avaient recours à des méthodes plus «personnelles» pour sélectionner les items lexicaux de leurs études. Une méthode couramment utilisée consistait, et consiste encore, à extraire ces items d'ouvrages scolaires en usage dans les niveaux scolaires étudiés.

Pendant longtemps, les chercheurs ont été dans l'impossibilité d'appréhender la base de connaissances lexicales des enfants. Seules quelques tentatives très locales avaient permis d'élaborer quelques bases de données (Le Vocabulaire des 
enfants des écoles de Genève, Prescott, 1929, cité par Ters et al., 1969), mais généralement trop restreintes pour être exploitées. On a alors souvent fait référence à des bases de données pédagogiques ( $L$ 'Échelle $D-B$ [Dubois-Buyse] et son adaptation le $V O B$ [Vocabulaire orthographique de base, Ters et al., 1969]). Les véritables progrès dans ce domaine viennent de la publication récente de bases de données pour enfants d'école primaire en français (NOVLEX et MANU$L E X)$. Par exemple, la base NOVLEX a été réalisée à partir d'ouvrages scolaires et extrascolaires de classe de CE2 et "peut être aussi exploitée pour des études avec des élèves de classes adjacentes » (Lambert et Chesnet, $2001: 280$ ). Elle offre, entre autres avantages, deux bases d'occurrences distinctes: l'une présentant la forme graphique de l'ensemble des mots analysés et fournissant ainsi la fréquence des formes fléchies des mots ; l'autre rapportant la fréquence cumulée des occurrences sur la racine.

\section{Expérience longitudinale}

\section{Problématique}

Malgré l'avancée importante que constitue l'arrivée de bases de données pour enfants, la sensibilité du phénomène étudié dans le cas de l'acquisition de l'accord nominal impose d'assurer un contrôle encore plus exigeant sur le matériel utilisé. On doit en effet s'assurer que les noms ont été «effectivement » rencontrés par les enfants, on doit connaitre le plus précisément possible la fréquence de cette rencontre ainsi que la modalité (singulier vs pluriel) sous laquelle cette rencontre a eu lieu. C'est ce que nous avons tenté de faire lors d'une étude longitudinale. Le principe retenu a été le suivant: nous avons sélectionné des noms rares dans un ensemble de bases de données théoriques pour les rendre ensuite expérimentalement fréquents ou rares, sous leurs différentes modalités de nombre (singulier vs pluriel) à différentes périodes de la scolarité d'enfants de $\mathrm{CP}$ et de CE1. Nous avons pu ainsi tester l'hypothèse de l'impact de la fréquence de rencontre des noms sur l'exactitude de l'accord en nombre. Cet effet devait 
se manifester, d'une part, par une facilitation à produire un accord pluriel sur les noms fréquemment rencontrés sous une modalité plurielle et, d'autre part, par une difficulté à produire l'accord singulier pour ces mêmes noms.

\section{Méthodologie}

\section{Participants}

Quatre vingt cinq enfants de CP (âge moyen 6;2 [6-6;11] en décembre de la première année scolaire) et 89 enfants de CE1 (âge moyen $7 ; 3[6 ; 11-7 ; 11]$ en décembre de la première année scolaire) ont participé à l'expérience, appariés sur leurs performances aux CPM de Raven (EAP, 1998) qui évaluent un niveau d'efficience non verbal et à l'épreuve $E 20$ de Khomsi (1990) qui évalue un niveau de lecture.

\section{Stimuli}

Quarante noms très peu fréquents, aisément imageables, ont été sélectionnés (noms d'animaux, de plantes ou d'objets: bonite, drave, colobe, sterne, dorine...) : absents de Novlex (2001), de Brulex (1990) et des livres scolaires utilisés par les enfants de l'expérience.

\section{Procédure}

Les participants ont été suivis pendant deux années de la manière suivante. Ils ont rencontré les items expérimentaux une première fois lors d'un prétest réalisé en décembre de la première année scolaire. Au cours de ce prétest, ils ont écrit sous dictée les 40 items expérimentaux. Les enfants ont ensuite participé à deux phases d'entrainement pendant lesquelles ils ont rencontré les items expérimentaux dans les conditions décrites ci dessous. Ils ont à nouveau été testés immédiatement après chaque phase d'entrainement (respectivement en mars puis en juin de la première année scolaire) puis, l'année scolaire suivante, à deux reprises: à la même période que celle du prétest (décembre) puis à la même période que celle du premier post test (mars).

Au cours d'une phase d'entrainement, les items expérimentaux étaient présentés en faisant varier les conditions de présentation. Une moitié d'entre eux était présentée au plu- 
riel, l'autre moitié au singulier. Une moitié était présentée fréquemment, l'autre, rarement. Les items étaient présentés précédés d'un article indéfini (un ou une pour le singulier; des pour le pluriel) et accompagnés de leur illustration (un exemplaire de l'animal, de la plante ou de l'objet pour les items présentés au singulier ou 3 exemplaires de ceux-ci pour les items présentés au pluriel). Les items fréquents faisaient l'objet, pendant 2 fois 10 semaines (les 2 trimestres de la scolarité suivant le prétest), d'un exercice de lecture collective bihebdomadaire (les items accompagnés de leurs illustrations étaient présentés et lus par l'enseignant) ainsi que d'un exercice de copie hebdomadaire, réalisé sur des carnets réservés à cet usage. Les items rares donnaient lieu à un exercice de copie une fois par trimestre. Ainsi, par période de 10 semaines, les items fréquents faisaient l'objet d'un exercice de lecture 20 fois et d'un exercice de copie 10 fois quand les items rares étaient copiés une fois.

La phase de test permettait de dicter les différentes catégories de noms, soit au singulier soit au pluriel. Les 40 noms expérimentaux étaient insérés en fin de phrases simples. Les enfants étaient munis de carnets de 40 pages, une page par item. La première partie de la phrase était écrite, y compris le déterminant, et il était demandé aux enfants d'écrire le nom manquant de la phrase dictée (Il ramasse une bonite; Il regarde des colobes).

\section{Résultats}

Les résultats rapportés concernent uniquement les noms dont la racine a été correctement orthographiée par les enfants. Ils sont analysés en deux temps. On étudie le pourcentage d'erreurs d'accord nominal d'abord pour les noms dictés au singulier puis pour les noms dictés au pluriel.

Une analyse globale a mis en évidence que le facteur «Type de session» interagissait avec plusieurs facteurs dont le niveau scolaire. Pour cette raison, nous rapportons directement ici les analyses niveau scolaire par niveau scolaire en étudiant session par session l'effet des facteurs «Fréquence de rencontre» et «Modalité de rencontre». 
Pour chaque type d'accord (singulier vs pluriel), les données ont fait l'objet d'une analyse de variance à 2 (fréquence de rencontre: fréquent; rare) $\times 2$ (modalité de rencontre: singulier; pluriel), avec mesures complètement répétées. Les analyses ont été conduites sur les participants (F1) ainsi que sur le matériel (F2).

\section{Accord singulier}

- Les données apparaissent dans le Tableau 1.

Tableau 1 - Pourcentages moyens d'erreurs d'accord de noms dictés au singulier en fonction du Niveau scolaire (CP;CE1), de la Session (mars 1 ; juin; décembre; mars 2), de la Fréquence de rencontre (fréquent; rare) et de la Modalité de rencontre (singulier; pluriel).

\begin{tabular}{|c|c|c|c|c|c|c|c|c|c|}
\hline & \multicolumn{4}{|c|}{ CP } & & \multicolumn{4}{|c|}{ CE1 } \\
\hline & \multicolumn{2}{|c|}{ Fréquent } & \multicolumn{2}{|c|}{ Rare } & & \multicolumn{2}{|c|}{ Fréquent } & \multicolumn{2}{|c|}{ Rare } \\
\hline & Sing. & Plurie & Sing. & Plurie & & Sing. & Plurie & Sing. & Plurie \\
\hline Mars 1 & 4.78 & 13 & 7.84 & 6.75 & Mars 1 & 2.28 & 8.4 & 6 & 3.93 \\
\hline$n=32$ & (11.85 & (27.38) & (23.15) & $(21.36)$ & $a=75$ & (9.33) & (15.14 & $(21.06)$ & $(12.56)$ \\
\hline Juin & 4.04 & 9.17 & 3.68 & 4.10 & Juin & 0.64 & 8.48 & 0.43 & 3.29 \\
\hline $\mathrm{n}=52$ & (15.87) & $(24.87)$ & (13.9) & (12.51) & $a=77$ & (4) & (15.69) & (3.79) & (13.16) \\
\hline Dé. & 3.89 & 10.56 & 4.40 & 4.40 & Dec. & 0.26 & 3.39 & 0.26 & 1.35 \\
\hline$n=53$ & (11.78) & (23.02) & $(17.03)$ & (16.31) & $\mathrm{n}=75$ & (2.29) & (9.38) & (2.29) & $(5.91)$ \\
\hline ars 2 & 1.87 & 4.28 & 2.21 & 5.86 & Mars 2 & 0.86 & 4.4 & 2.31 & 2.46 \\
\hline$n=58$ & $(8.4)$ & (15.65) & $(10.96)$ & $\{19.89\}$ & $n=70$ & (4.3) & $(11.8)$ & (8.4) & $(9.6)$ \\
\hline
\end{tabular}

Au CP, l'interaction Fréquence * Modalité n'est significative qu'en mars $1: \mathrm{F} 1(1,31)=4.69, \mathrm{p}=.038$ et en décembre: $\mathrm{F} 1$ $(1,52)=4.25, p=.044$. Dans les deux cas, l'effet de la modalité ne se manifeste que pour les noms fréquents (respectivement: marginalement, $\mathrm{F} 1(1,31)=3.68, \mathrm{p}=.06 ; \mathrm{F} 1$ $(1,52)=5.29, \mathrm{p}=.025)$ et va dans le sens attendu (davantage d'erreurs pour accorder au singulier un nom fréquemment rencontré au pluriel). 
Au CE1, l'interaction Fréquence * Modalité n'est significative que pour les deux premières sessions: mars 1: F1 $(1,74)=7.23, p=.009$ et juin: $F 1(1,76)=6.09, p=.016$. Ici encore, dans les deux cas, l'effet de la modalité ne se manifeste que pour les noms fréquents (respectivement: F1 $(1,74)=10.38, \mathrm{p}=.002 ; \mathrm{F} 1(1,76)=18.16, \mathrm{p}=.0001)$ et va à nouveau dans le sens attendu.

\section{Accord pluriel}

- Les données apparaissent dans le Tableau 2.

Tableau 2 - Pourcentages moyens d'erreurs d'accord de noms dictés au pluriel en fonction du Niveau scolaire (CP; CE1), de la Session (mars 1 ; juin ; décembre; mars 2), de la Fréquence de rencontre (fréquent; rare) et de la Modalité de rencontre (singulier; pluriel). Les écarts-types figurent entre parenthèses.

\begin{tabular}{|c|c|c|c|c|c|c|c|c|c|}
\hline & \multicolumn{4}{|c|}{$\mathrm{CP}$} & & \multicolumn{4}{|c|}{$\mathrm{CEl}$} \\
\hline & \multicolumn{2}{|c|}{ Friézuent } & \multicolumn{2}{|c|}{ Rare } & & \multicolumn{2}{|c|}{ Fréquent } & \multicolumn{2}{|c|}{ Rare } \\
\hline & Sing. & Pluriel & Sing. & Pluniel & & Sing. & Plurie & Sing. & Pluniel \\
\hline Mars 1 & 73.78 & 50.04 & 61.97 & 55.37 & Man 1 & 52.31 & 37.2 & 51.22 & 44.95 \\
\hline $1=32$ & (33.26) & (10.22) & (335.9) & (43.73) & $1 \times 75$ & (32.27) & $(33.66)$ & (39.04) & (39.02) \\
\hline Juin & 66.22 & 48.97 & 68.91 & 66.09 & Juin & 53.37 & 38.33 & 55.26 & 52.77 \\
\hline$z=52$ & (38.46) & (36.03) & (33.65) & (40.84) & $n=\pi$ & (34.99) & (39) & $(39.61)$ & (3829) \\
\hline DEC. & 61 & 50.78 & 56.25 & 56.91 & DES. & 45 & 36.97 & 43.57 & 41.25 \\
\hline$a=93$ & (37.69) & (39.7) & (40.91) & (41.85) & $\theta=75$ & (39.7) & (38.8) & (4l.4) & $(42.8)$ \\
\hline Mars 2 & 55.2 & 51.61 & 58.02 & 54.02 & Mars 2 & 34.51 & 30.13 & 32.89 & 29.64 \\
\hline$a=58$ & $(40.7)$ & $(41.66)$ & $(40.9)$ & (41.33) & $g=70$ & (39.3) & (38.9) & (36.4) & (35.8) \\
\hline
\end{tabular}

$\mathrm{Au} \mathrm{CP}$, l'interaction Fréquence*Modalité est significative lors des seules trois premières sessions: mars 1: F1 $(1,31)=4.17, \mathrm{p}=.049$, juin: $\mathrm{F} 1(1,51)=5.52, \mathrm{p}=.023$, et décembre: $\mathrm{F} 1(1,52)=4.06, \mathrm{p}=.04$. Dans les trois cas, l'effet de la modalité ne se manifeste que pour les noms fréquents (respectivement: $\mathrm{F} 1(1,31)=15.5, \mathrm{p}=.0001 ; \mathrm{F} 1$ $(1,51)=14.33, \mathrm{p}=.0004$ mais $\mathrm{F} 2(1,39)<1$, ns.; F1 $(1,52)=6.52, \mathrm{p}=.013)$ et va systématiquement dans le sens 
attendu (une facilitation de l'accord pluriel pour les noms fréquemment rencontrés au pluriel).

Au CE1, l'interaction Fréquence * Modalité est marginalement significative en session de mars $1: F 1(1,74)=3.15, \mathrm{p}=$ .07 puis n'est plus significative qu'en juin: $F 1(1,76)=9.6, p$ $=.003$. Dans les deux cas, ici encore, l'effet de la modalité ne se manifeste que pour les noms fréquents (respectivement : F1 $(1,74)=21.19, \mathrm{p}=.0001 ; \mathrm{F} 1(1,76)=20.01, \mathrm{p}=.0001)$ et $\mathrm{va}$ systématiquement dans le sens attendu.

\section{Discussion}

L'objectif de cette recherche était de vérifier que la mise en œuvre d'une règle orthographique simple (accorder un nom en nombre) ne relevait pas exclusivement de l'application consciente d'une règle. En effet, pendant longtemps, pédagogues, rééducateurs et psychologues se sont accommodés d'une telle conception: la production écrite s'enseigne et la maitrise de sa dimension orthographique est sous-tendue par l'apprentissage de règles. Sans doute parce que nous sommes tous d'anciens élèves, nous est-il difficile de concevoir que l'écrit s'acquiert autrement que de la manière dont on nous l'a enseigné.

Si l'acquisition de la morphologie flexionnelle du nombre nominal relevait exclusivement de l'application d'une règle, les performances de l'enfant devraient respecter deux principes.

D'une part, elles devraient évoluer ainsi: 1) non marquage systématique du pluriel (avant la présentation de la règle);2) marquage occasionnel du pluriel (en cours d'apprentissage de la règle); 3) marquage systématique du pluriel (une fois la règle maitrisée). Ces étapes ont souvent été observées lors de travaux sur la production de la morphologie du nombre en français (Fayol, Hupet et Largy, 1999) confortant la thèse d'un apprentissage conscient, long et cognitivement couteux, relevant de l'application d'une règle algorithmique (« $\underline{\text { si nom }}$ pluriel, alors lui ajouter $-s »)$.

D'autre part, tous les noms (de même complexité lexicale) devraient être traités de la même manière au cours de ces 3 
étapes. Or, des travaux récents, focalisés sur l'étude de la troisième étape, celle de l'expertise, ont montré que les élèves du cycle 3 de l'école primaire et de collège accordaient mieux au pluriel certains noms que d'autres noms. Ce résultat a conduit Cousin et al. (2002) à considérer que la production de la morphologie du nombre nominal ne relevait pas exclusivement de la mise en œuvre d'une règle mais interférait avec l'activation en mémoire d'une forme globale du nom incluant la flexion à laquelle il est habituellement associé.

Tester une telle hypothèse supposait d'opérer un contrôle minutieux sur les variables manipulées. Il s'agissait de s'assurer des noms précis effectivement rencontrés par les enfants, de la flexion associée à chaque nom lors de la rencontre et de la fréquence avec laquelle ces noms étaient rencontrés. C'est ce que nous avons fait en demandant à des enfants de $\mathrm{CP}$ et de CE1 de produire ces noms au singulier et au pluriel, dans le cadre d'une étude longitudinale. Le choix de ces deux niveaux d'âge (CP et $\mathrm{CE} 1$ ) permettait cette fois d'étudier l'impact de la fréquence de rencontre des noms et de la modalité de nombre sous laquelle ils avaient été rencontrés, dans deux situations différentes : avant (CP) et après (CE1) la présentation de la règle d'accord du nom.

Les résultats de cette étude montrent clairement que la forme flexionnelle (singulier vs pluriel) dominante du nom interfère avec la qualité de l'accord produit. Au CP, les enfants (1) accordent mieux au pluriel les noms qu'ils ont précédemment rencontrés ainsi fléchis et (2) produisent moins bien au singulier les noms précédemment rencontrés au pluriel. De plus, le phénomène résiste à l'enseignement de la règle. En effet, on retrouve chez les élèves de CE1 les effets (1) et (2) précédemment évoqués. Ce résultat montre que, même lors de la phase de gestion algorithmique de l'accord (cf. supra), tous les noms ne sont pas traités de la même façon. Au contraire, tout se passe comme si le fait d'avoir précédemment rencontré un nom fléchi au pluriel avait une incidence notable sur la production ultérieure de l'accord de ce nom.

La fréquence de rencontre des noms joue ainsi un rôle non négligeable dans la gestion de l'accord. Son impact apparait à travers l'interaction entre ce facteur et la modalité de nombre sous laquelle les noms sont rencontrés. En effet, les effets (1 
et 2) précédemment évoqués ne s'observent que dans la situation des noms fréquemment rencontrés. Ce résultat est compatible avec l'hypothèse d'un stockage d'une forme globale du nom pluriel incluant le -s final.

Un autre résultat confirme l'impact de la fréquence de rencontre: les effets 1 et 2 ne s'observent qu'immédiatement après les périodes d'entrainement (ou presque: cf. ci-dessous le cas du CP). On n'observe aucun effet en mars de la deuxième année, quelle que soit la variable dépendante (accorder au singulier ou accorder au pluriel). Ainsi l'impact de la rencontre répétée d'un même nom sous sa forme plurielle sur la production ultérieure de l'accord apparait d'une durée limitée. Une différence importante nécessite toutefois d'être notée: cet impact parait plus persistant chez l'enfant jeune. En effet, alors qu'il disparait systématiquement dès décembre (cf. Tableaux 1 et 2) chez les ex-CE1, il ne disparait qu'en mars de la deuxième année chez les ex-CP. Peut-être sommes-nous ici en présence d'un effet d'âge d'acquisition (AOA : Bonin, Fayol et Chalard, 2001)? Il se pourrait que l'enfant de $6 / 7$ ans mémorise de manière plus stable et/ou plus durable l'enveloppe orthographique globale des mots que ne le ferait un enfant plus âgé. Il se pourrait aussi que les enfants plus âgés bénéficient, pour l'analyse et la mise en œuvre de la morphologie flexionnelle du nombre, de pratiques métalinguistiques plus étendues. De futures recherches devront $\mathrm{s}$ 'attacher à tester ces hypothèses.

\section{Références bibliographiques}

BECK, M. (1995): Tracking down the source of NS-NNS differences in syntactic competence, Language and Cognitive Processes, 10, 425-455.

Bonin, P., Fayol, M. et Chalard, M. (2001): Age of acquisition and word frequency in written picture naming, The Quarterly Journal of Experimental Psychology, 54A, 469-489.

Braine, M.D.S., BRody, R. E, Brooks, P.J., Sudhalter, V., Ross, J.A., Catalano, L. et Fisch, S.M. (1990): Exploring language acquisition in children with a miniature artificial language: Effects of item and pattern frequency, arbitrary subclasses, and correction, Journal of Memory and Language, 29, 591-610. 
Brissaud, C. (1999a): L'infinitif en -er: Difficultés orthographiques au collège, La Linguistique, 35, 61-78.

BrisSAUD, C. (1999b): La réalisation de l'accord du participe passé employé avec «avoir». De l'influence de quelques variables linguistiques et sociales, Langage et Société, 88, 5-24.

Content, A., Mousty, P. et Radeau, M. (1990): Brulex : une base de données lexicales informatisée pour le français écrit et parlé, L'Année psychologique, 90, 551-566.

Cousın, M.P. (2000): Acquisition des marques écrites du pluriel nominal et verbal: le rôle de l'apprentissage implicite, DEA de Psychologie, Université de Rouen.

Cousin, M.P., LARGY, P. et FAyOL, M. (2002): Sometimes early learned instances interfere with the implementation of rules: The case of nominal number agreement, Current Psychology Letters : Behavior, Brain et Cognition, 2-8, 51-65.

Cousin, M.P., LARgY, P. et FAyOL, M. (2003) : Produire la morphologie flexionnelle du nombre nominal: Etude chez l'enfant d'école primaire, Rééducation Orthophonique, 213, 115-129.

DuBoIs, J. (1965): Grammaire structurale du français: nom et pronom, Paris, Larousse.

DÉDÉyAN, A. et LARGY, P. (2003): Réviser la morphologie flexionnelle verbale: Etude chez l'enfant et l'adulte, Rééducation Orthophonique, 213, 97-113.

E.A.P. (1998): CPM de Raven, Paris.

Ellis, N.C. et SCHMIDT, R. (1998): Rules or associations in the acquisition of morphology? The frequency by regularity interaction in human and PDP learning of morphosyntax, Language and Cognitive Processes, 13, 307-336.

Fayol, M., Hupet, M., Largy, P. (1999): The acquisition of subject-verb agreement in written french: From novices to experts, Reading and Writing: An interdisciplinarity Journal, 11, 153174.

IMBS, P. (1971): Etudes statistiques sur le vocabulaire français. Dictionnaire des fréquences. Vocabulaire littéraire des XIXe et $X X^{\top}$ siècles, Centre de recherche pour un trésor de la langue française (CNRS), Nancy, Paris, Librairie Marcel-Didier.

KHOMSI, A. (1990): E20, Editions du centre de psychologie appliquée, Paris.

LAMBERT, E. et CHeSNeT, D. (2001): Novlex: une base de données lexicales pour les élèves de primaire, L'Année psychologique, $101,277-288$.

LARGY, P. (2001): La révision des accords nominal et verbal chez I'enfant, L'Année psychologique, 101, 221-245. 
Largy, P., Chanquoy, L. et Dédéyan, A. (2004): Orthographic revision: The case of subject-verb agreement in French. In L. Allal, L. Chanquoy et P. Largy (Eds.), Revision: Cognitive and Instructional Processes, Dordrecht: Kluwer, 39-62.

LARGY, P. et DÉDEYAN, A. (2002): Automatisme en détection d'erreurs d'accord sujet-verbe: étude chez l'enfant et l'adulte, L'Année Psychologique, 102, 201-234.

LARGY, P., DÉDÉYAN, A., Hupet, M. (in press): Orthographic revision: A developmental study of how novice and expert writers detect subject-verb agreement errors in written texts, British Journal of Educational Psychology.

TERs, F., MAYER, G. et ReICHENBACH, D. (1969): L'Echelle D-B d'orthographe usuelle française: Vocabulaire orthographique de base, Messeiller, Neuchâtel.

Thibault, M.-P. (dir. 2003): Langage écrit: morphologie et conscience morphologique, Rééducation Orthophonique, 213.

Totereau, C., Barrouillet, P. et Fayol, M. (1998): Overgeneralizations of number inflections in the learning of written French: The case of noun and verb, British Journal of Developmental Psychology, 16, 447-464.

Totereau, C., Thévenin, M.-G. et Fayol, M. (1997): The development of the understanding of number morphology in French. In C. Perfetti, M. Fayol et L. Rieben (Eds.), Learning to spell, HilIsdale : L.E.A., 97-114.

YANG, L.R. et GivoN, T. (1997) : Benefits and drawbacks of controlled laboratory studies of second language acquisition: The Keck second language learning project, Studies in Second Language Acquisition, 19, 173-194.

Zeno, S.M., Ivens, S.H., Mrllard, R.T. et Duvvuri, R. (1995): The educator's word frequency guide, Cleveland, Touchstone Applied Science Associates. 